\title{
CONTROL-ORIENTED MODEL OF AN \\ INTEGRATED FUEL CELL STACK AND FUEL PROCESSOR SYSTEM ${ }^{1}$
}

\author{
Jay T. Pukrushpan* Anna G. Stefanopoulou** \\ Subbarao Varigonda ${ }^{* * *}$ \\ * University of Michigan, Ann Arbor, MI \\ ** University of Michigan, Ann Arbor, MI \\ *** United Technologies Research Center, Hartford, CT
}

\begin{abstract}
A control-oriented dynamic model of a catalytic partial oxidation-based fuel processor is developed using physics-based principles. The fuel processor converts a hydrocarbon fuel to a hydrogen $\left(\mathrm{H}_{2}\right)$ rich mixture that is directly feed to the PEM-FC. Cost and performance requirements of the total powertrain typically lead to highly integrated designs and stringent control objectives. Physics based component models are extremely useful in understanding the system level interactions, implications on system performance and in model-based controller design. The model can be used in a multivariable analysis to determine characteristics of the system that might limit performance of a controller or a control design. The model can also be used to assist in measurement selection and to develop a plant observer to predict or estimate critical plant variables and conditions.
\end{abstract}

Keywords: Fuel Cell, Fuel Processor, Multivariable Feedback, Linear Control, Partial Oxidation

\section{INTRODUCTION}

Inadequate infrastructure for hydrogen refueling, distribution, and storage makes fuel processor technology an important part of the fuel cell system. Methanol, gasoline, and natural gas are examples of fuels being considered as fuel cell energy sources. Different processes involved in converting carbonbased fuel to hydrogen are presented in (Birch, 2001; Brown, 2001).

For large commercial vehicles and residential applications, fueling the fuel cell system using natural gas is often preferred because of its wide availability and extended distribution system (Dicks, 1996). Common methods of converting natural gas to hydrogen include steam reforming and partial oxidation. The most common method, steam reforming, which is endothermic, is well suited for steady-state operation and can deliver a relatively high concentration of hydrogen (Ahmed and Krumpelt, 2001), but it suffers from a poor transient operation (Brown, 2001). On the other hand, the partial oxidation offers several other advantages such as compactness, rapid-startup,

\footnotetext{
1 Supported by the National Science Foundation under contracts CMS-0201332 and CMS-0219623, United Technologies Research Center and a fellowship from the Royal Thai Government
}

and responsiveness to load changes (Dicks, 1996), but delivers lower conversion efficiency.

The main reactor of a partial oxidation-based natural gas fuel processing system (FPS) is a catalytic partial oxidation (CPOX) reactor. Here, hydrogen-rich gas is produced by mixing natural gas with air over a catalyst bed. The amount of hydrogen created in the FPS depends on both the catalyst bed temperature and the ratio between the supply rate of $\mathrm{CH}_{4}$ and the CPOX air to fuel ratio, more specifically, the oxygen to carbon ratio. This oxygen to carbon ratio also influences the amount of heat generated in the CPOX, which then affects the CPOX catalyst bed temperature.

System level dynamic models of fuel cell power plants built from physics-based component models are extremely useful in understanding the system level interactions, implications for system performance and model-aided controller design. The system level dynamic models also help in evaluating alternative system architectures in an integrated design and control paradigm. In this paper, we present a dynamic model for the FPS control of the air blower and the fuel valve for fast and efficient $\mathrm{H}_{2}$ generation. More specifically, we concentrate on the dynamics associated with two main control objectives. First, to prevent stack 
$\mathrm{H}_{2}$ starvation, which can permanently damage the stack (Song et al., 2000), the hydrogen flow from the FPS must respond rapidly and robustly to changes in stack power level, i.e. changes in stack current (Pukrushpan et al., 2002). Unfortunately, oversupply of $\mathrm{H}_{2}$ by adjusting the FPS flow at a higher steadystate level is not an option because this will cause wasted hydrogen from the anode exhaust (Song et al., 2000). Thus, hydrogen generation needs to follow the current load in a precise and fast manner. Second, the temperature of the CPOX must be maintained at a certain point. Exposure to high temperature will permanently damage the CPOX catalyst bed while low CPOX temperature slows down the fuel reaction rate (Zhu et al., 2001). The optimization of these goals during transient operations can be achieved by coordinating CPOX air blower command and the fuel (natural gas) valve command.

We neglect variations of the pressure, concentration and temperature within various system stages and lump them into spatially averaged variables and can be described using ordinary differential equations. The model is parameterized and validated against the results from a high-order fuel cell system model (Eborn et al., 2003). Then, we present an observability analysis of the model that helps in selecting cost effective sensors for plant estimation.

\section{OVERVIEW OF THE FUEL PROCESSING SYSTEM (FPS)}

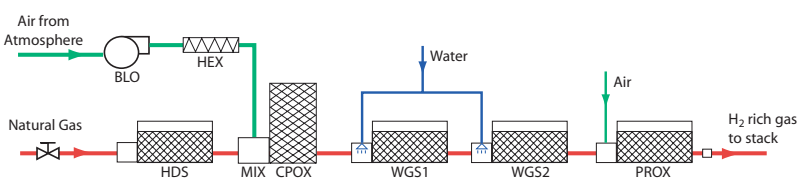

Fig. 1. FPS components

Figure 1 illustrates the components in a natural gas fuel processing system (FPS) (Thomas et al., 2000). The FPS is composed of four main reactors, namely, hydro-desulfurizer (HDS), catalytic partial oxidation (CPOX), water gas shift (WGS), and preferential oxidation (PROX). Natural gas (Methane $\mathrm{CH}_{4}$ ) is supplied to the FPS from either a high-pressure tank or a high-pressure pipeline. Sulfur, which poisons the water gas shift catalyst (Brown, 2001), is then removed from the natural gas stream in the HDS (Dicks, 1996; Gardner et al., 2002). The main air flow is supplied to the system by a blower (BLO) which draws air from the atmosphere. The air is then heated in the heat exchanger (HEX). The heated air and the de-sulfurized natural gas stream are then mixed in the mixer (MIX). The mixture is then passed through the catalyst bed inside the catalytic partial oxidizer (CPOX) where $\mathrm{CH}_{4}$ reacts with oxygen to produce $\mathrm{H}_{2}$. There are two main chemical reactions taking place in the CPOX: partial oxidation (POX) and total oxidation (TOX) (Zhu et al., 2001; Larentis et al., 2001):

$$
\begin{array}{ll}
(\mathrm{POX}) & \mathrm{CH}_{4}+\frac{1}{2} \mathrm{O}_{2} \rightarrow \mathrm{CO}+2 \mathrm{H}_{2} \\
(\mathrm{TOX}) & \mathrm{CH}_{4}+2 \mathrm{O}_{2} \rightarrow \mathrm{CO}_{2}+2 \mathrm{H}_{2} \mathrm{O}
\end{array}
$$

Heat is released from both reactions. However, TOX reaction releases more heat than POX reaction. Hydrogen is created only in POX reaction and, therefore, it is preferable to promote this reaction in the CPOX. However, carbon monoxide (CO) is also created along with $\mathrm{H}_{2}$ in the $\mathrm{POX}$ reaction as can be seen in (1). Since CO poisons the fuel cell catalyst, it is eliminated using both the water gas shift converter (WGS) and the preferential oxidizer (PROX). As illustrated in Figure 1, there are typically two WGS reactors operating at different temperatures (Brown, 2001; Ledjeff-Hey et al., 2000). In the WGS, water is injected into the gas flow in order to promote a water gas shift reaction:

$$
(W G S) \quad \mathrm{CO}+\mathrm{H}_{2} \mathrm{O} \rightarrow \mathrm{CO}_{2}+\mathrm{H}_{2}
$$

Note that even though the objective of WGS is to eliminate CO, hydrogen is also created from the WGS reaction. The level of $\mathrm{CO}$ in the gas stream after WGS is normally still high for fuel cell operation and thus oxygen is injected (in the form of air) into the PROX reactor to react with the remaining $\mathrm{CO}$ :

$$
(\mathrm{PROX}) \quad 2 \mathrm{CO}+\mathrm{O}_{2} \rightarrow 2 \mathrm{CO}_{2}
$$

The amount of air injected into the PROX is typically twice the amount that is needed to maintain the stoichiometric reaction in (4) (Brown, 2001; Doss et al., 2001).

\section{CONTROL-ORIENTED FPS MODEL}

The FPS model is developed with a focus on the dynamic behaviors associated with the flows and pressures in the FPS and also the temperature of the CPOX.

\subsection{Modeling Assumptions}

Several assumptions are made in order to simplify the FPS model. Since the control of WGS and PROX reactants are not studied, the two components are lumped together as one volume and the combined volume is called WROX (WGS+PROX). It is also assumed that both components are perfectly controlled such that the desired values of the reactants are supplied to the reactors. The de-sulfurization process in the HDS is not modeled and thus the HDS is viewed as a storage volume. It is assumed that the composition of the air entering the blower is constant. Additionally, any temperature other than the CPOX temperature is assumed constant and the effect of temperature changes on the pressure dynamics is assumed negligible. The volume of CPOX is relatively small and is thus ignored. It is also assumed that the CPOX reaction is rapid and reaches equilibrium before the flow exit the CPOX reactor. Finally, all 
gases obey the ideal gas law and all gas mixtures are perfect mixtures. Figure 2 illustrates the simplified system and state variables used in the model.

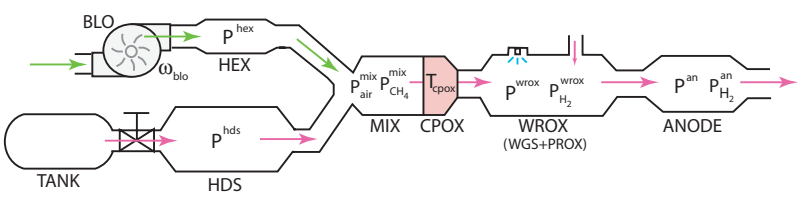

Fig. 2. FPS dynamic model

\subsection{Model States}

The dynamic states in the model, shown also in Figure 2 , are blower speed, $\omega_{b l o}$, heat exchanger pressure, $p^{h e x}$, HDS pressure, $p^{h d s}$, mixer $\mathrm{CH}_{4}$ partial pressure, $p_{\mathrm{CH}_{4}}^{m i x}$, mixer air partial pressure, $p_{\text {air }}^{m i x}$, CPOX temperature, $T_{c p x}$, WROX (combined WGS and PROX) volume pressure, $p^{w r x}$, WROX hydrogen partial pressure, $p_{H_{2}}^{w r x}$, anode pressure, $p^{a n}$, and anode hydrogen partial pressure, $p_{\mathrm{H}_{2}}^{a n}$.

\subsection{Orifice}

The flow between any two volumes in the FPS system is based on the orifice flow equation. Specifically, the mass flow rate between two volumes is given as a function of upstream pressure, $p_{1}$, and downstream pressure, $p_{2}$. The flow is assumed turbulent and the rate is governed by

$$
W=W_{0} \sqrt{\frac{p_{1}-p_{2}}{\Delta p_{0}}}
$$

where $W_{0}$ and $\Delta p_{0}$ are the nominal air flow rate and the nominal pressure drop of the orifice, respectively.

\subsection{Blower (BLO)}

The speed of the blower is modeled as a first-order dynamic system with time constant $\tau_{b}$. The governing equation is

$$
\frac{d \omega_{b l o}}{d t}=\frac{1}{\tau_{b}}\left(\frac{u_{b l o}}{100} \omega_{0}-\omega_{b l o}\right)
$$

where $u_{b l o}$ is the blower command signal (range between 0 and 100) and $\omega_{0}$ is the nominal blower speed $(3600 \mathrm{rpm})$. The gas flow rate through the blower, $W_{b l o}$, is determined using the blower map. The blower time constant is 0.3 seconds.

\subsection{Heat Exchanger Volume (HEX)}

The only dynamics considered in the heat exchanger is the pressure dynamics. The changes in temperature of the gas are ignored and it is assumed that the effects of actual temperature changes on the pressure dynamics are negligible. The rate of change in air pressure of the HEX is described by

$$
\frac{d p^{h e x}}{d t}=\frac{R T_{h e x}}{M_{\text {air }}^{a t m} V_{\text {hex }}}\left(W^{\text {blo }}-W^{\text {hex }}\right)
$$

where $M_{\text {air }}^{\text {atm }}$ is the molecular weight of the air flow through the blower.

\subsection{Hydro-Desulfurizer Volume (HDS)}

The pressure of the gas in the HDS is governed by the mass balance principle. It is assumed that the natural gas fed to the HDS is pure methane $\left(\mathrm{CH}_{4}\right)$ (Brown, 2001), and thus the desulfurization process is not modeled. The HDS is then considered as a gas volume and the pressure changes are modeled by

$$
\frac{d p^{h d s}}{d t}=\frac{R T_{h d s}}{M_{C H_{4}} V_{h d s}}\left(W_{f u e l}-W^{h d s}\right)
$$

where $W^{\text {hds }}$ is the rate of mass flow from HDS to the mixer (MIX), and is calculated as a function of $p^{h d s}$ and $p^{m i x}$ using the orifice equation (5). The temperature of the gas, $T_{h d s}$, is assumed constant.

The flow rate of methane into the HDS, $W_{f u e l}$, is controlled by a fuel valve. The orifice equation (5) with variable gain based on the valve input signal, $u_{\text {valve }}(0$ to 100), is used to model the flow through the valve.

$$
W_{\text {fuel }}=\left(\frac{u_{\text {valve }}}{100}\right) W_{0, \text { valve }} \sqrt{\frac{p^{\text {tank }}-p^{h d s}}{\Delta p_{0, \text { valve }}}}
$$

where $p^{\text {tank }}$ is the fuel tank or supply line pressure.

\subsection{Mixer $(M I X)$}

The natural gas flow from the HDS and the air flow from the blower are combined in the mixer (MIX). Two dynamic variables in the mixer model are the methane pressure, $p_{C_{4}}^{m i x}$, and the air pressure, $p_{a i r}^{m i x}$. The state equations of the MIX model are

$$
\begin{aligned}
& \frac{d p_{C H_{4}}^{m i x}}{d t}=\frac{R T_{m i x}}{M_{C H_{4}} V_{m i x}}\left(W^{h d s}-x_{C H_{4}}^{m i x} W^{c p x}\right) \\
& \frac{d p_{a i x}^{m i x}}{d t}=\frac{R T_{m i x}}{M_{a i r}^{a m m} V_{m i x}}\left(W^{h e x}-x_{a i r}^{m i x} W^{c p x}\right)
\end{aligned}
$$

where $W^{c p x}$ is the flow rate through the CPOX which is calculated as a function of $p^{m i x}$ and $p^{w r x}$. The mixer total pressure is the sum of the $\mathrm{CH}_{4}$ and the air pressures, $p^{m i x}=p_{C_{4}}^{m i x}+p_{a i r}^{m i x}$. Based on $p_{C_{H_{4}}^{m i x}}^{m i x}$ and $p_{\text {air }}^{\text {mix }}$, the mass fractions of $\mathrm{CH}_{4}$ and the air in the mixer, $x_{\mathrm{CH}_{4}}^{m i x}$ and $x_{a i r}^{m i x}$, are calculated by

$$
x_{C H_{4}}^{m i x}=\frac{1}{1+\frac{M_{a i r}^{a t m}}{M_{C H_{4}}} \frac{p_{\text {aix }}^{m i x}}{p_{C H_{4}}^{m i x}}} ; \quad x_{a i r}^{m i x}=1-x_{C H_{4}}^{m i x}
$$

where $M_{C_{4}}$ and $M_{a i r}^{a t m}$ are the molar masses of methane and atmospheric air, respectively. The temperature of the mixer gas, $T_{m i x}$, is assumed constant. 
The mass fractions of nitrogen, oxygen and vapor in the mixer needed for the calculation of the CPOX reactions are $x_{i}^{m i x}=x_{i}^{a t m} x_{a i r}^{m i x}$ where $i$ represents $\mathrm{N}_{2}$, $\mathrm{O}_{2}$ and $\mathrm{H}_{2} \mathrm{O}$. The oxygen to carbon, i.e., $\mathrm{O}_{2}$ to $\mathrm{CH}_{4}$, (mole) ratio, $\lambda_{O_{2 C}}$, which influences the reaction rate in the CPOX, is calculated by

$$
\lambda_{\mathrm{O}_{2 C}}=y_{\mathrm{O}_{2}}^{a t m} \frac{p_{a i r}^{m i x}}{p_{C H_{4}}^{m i x}}
$$

where $y_{\mathrm{O}_{2}}^{\text {atm }}$ is the oxygen mole fraction of the atmospheric air.

\subsection{Catalytic Partial Oxidation (CPOX)}

The only dynamics considered in the CPOX is the catalyst temperature, $T_{c p x}$. The temperature dynamics is modeled using energy balance equation

$m_{\text {bed }}^{c p x} C_{P, \text { bed }}^{c p x} \frac{d T_{c p x}}{d t}=\left[\begin{array}{c}\text { inlet en- } \\ \text { thalpy } \\ \text { flow }\end{array}\right]-\left[\begin{array}{c}\text { outlet } \\ \text { enthalpy } \\ \text { flow }\end{array}\right]+\left[\begin{array}{c}\text { heat } \\ \text { from } \\ \text { reaction }\end{array}\right]$

where $m_{\text {bed }}^{c p x}(\mathrm{~kg})$ and $C_{P, \text { bed }}^{c p x}(\mathrm{~J} / \mathrm{kg} \cdot \mathrm{K})$ are mass and specific heat capacity of the catalyst bed, respectively. The last two terms on the right hand side of (14) depend on the reaction taking place in the CPOX.

In the catalytic partial oxidation reactor, methane $\mathrm{CH}_{4}$ is oxidized to produce hydrogen. There are two $\mathrm{CH}_{4}$ oxidation reactions: partial oxidation (POX) and total oxidation (TOX). The other two secondary reactions considered here are water formation, or hydrogen oxidation $\left(2 \mathrm{H}_{2}+\mathrm{O}_{2} \rightarrow 2 \mathrm{H}_{2} \mathrm{O}\right)$, and carbon monoxide preferential oxidation $\left(2 \mathrm{CO}+\mathrm{O}_{2} \rightarrow\right.$ $2 \mathrm{CO}_{2}$ ). The species entering the CPOX include $\mathrm{CH}_{4}$, $\mathrm{O}_{2}, \mathrm{H}_{2} \mathrm{O}$, and $\mathrm{N}_{2}$. Nitrogen does not react in the CPOX. The water may react with $\mathrm{CH}_{4}$ through steam reforming reaction (Brown, 2001); however, this reaction is ignored in this study. Methane reacts with oxygen to create the final product, which contains $\mathrm{H}_{2}, \mathrm{H}_{2} \mathrm{O}, \mathrm{CO}, \mathrm{CO}_{2}, \mathrm{CH}_{4}$, and $\mathrm{O}_{2}$ ( $\mathrm{Zhu}$ et al., 2001). The amount of each species depends on the initial oxygen to carbon $\left(\mathrm{O}_{2}\right.$ to $\left.\mathrm{CH}_{4}\right)$ ratio, $\lambda_{\mathrm{O}_{2 C} \mathrm{C}}$, of the reactants and the temperature of the CPOX catalyst bed, $T_{c p x}$. A set of equations is developed to calculate the conversion of the gases in CPOX is based on the reactions in (1) and (2) and the thermodynamic equilibrium analysis in (Zhu et al., 2001). A plot of products calculated, assuming no inlet $\mathrm{N}_{2}$ and $\mathrm{H}_{2} \mathrm{O}$, is shown in Figure 3. Further details of the model are presented in (Pukrushpan, 2003).

\subsection{Water Gas Shift Converter and Preferential Oxidation Reactor (WROX)}

The water gas shift converter and the preferential oxidation reactor are lumped together as one volume, denoted as WROX. Three flows entering the volume are $\mathrm{H}_{2}$-rich gas flow from the CPOX, $W^{c p x}$, water injection needed for WGS reaction, $W_{\mathrm{H}_{2} \mathrm{O}}^{w g s}$, and air injection required for PROX reaction, $W_{\text {air }}^{\text {prox }}$. The
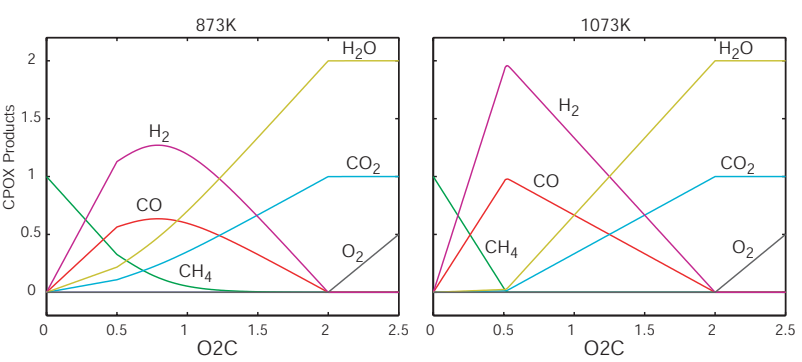

Fig. 3. Products of CPOX reaction per unit of $\mathrm{CH}_{4}$ entering CPOX

WROX model has two states, which are total pressure, $p^{w r x}$, and hydrogen pressure, $p_{H_{2}}^{w r x}$. Since the amount of $\mathrm{CO}$ created in CPOX is proportional to the rate of $\mathrm{H}_{2}$ created ( $\mathrm{POX}$ reaction), it is assumed that the rate of $\mathrm{H}_{2}$ generated in the WGS is a fixed percentage $\left(\eta_{w r x}\right)$ of the rate of hydrogen generated in the CPOX. The state equations are

$\frac{d p^{w r x}}{d t}=\frac{R T_{w r x}}{M_{w r x} V_{w r x}}\left(W^{c p x}-W^{w r x}+W_{H_{2} O}^{w g s}+W_{a i r}^{p r o x}\right)$

$\frac{d p_{H_{2}}^{w r x}}{d t}=\frac{R T_{w r x}}{M_{H_{2}} V_{w r x}}\left(\left(1+\eta_{w r x}\right) W_{H_{2}}^{c p x}-x_{H_{2}}^{w r x} W^{w r x}\right)$

where $M_{w r x}$ is an average molecular weight of the gas in WROX, and $T_{w r x}$ is an average temperature of WGSs and PROX. The WROX exit flow rate, $W^{w r x}$, is calculated using the nozzle equation (5) based on the pressure drop between WROX and anode volume, $p^{w r x}-p^{a n}$. The hydrogen mass fraction in WROX, $x_{H_{2}}^{w r x}$, can be determined from the two states by

$$
x_{H_{2}}^{w r x}=\frac{M_{H_{2}}}{M_{w r x}} \frac{p_{H_{2}}^{w r x}}{p^{w r x}}
$$

The rate of water injected into WROX, $W_{\mathrm{H}_{2} \mathrm{O}}^{w g}$, is equal to the amount that is required to cool the gas from CPOX down to the desired WGSs inlet temperatures (Brown, 2001; Doss et al., 2001). There are two WGS reactors and thus the total rate of water injected is $W_{\mathrm{H}_{2} \mathrm{O}}^{w g s}=W_{\mathrm{H}_{2} \mathrm{O}}^{w g s 1}+W_{\mathrm{H}_{2} \mathrm{O}}^{w g s 1}$. The flow rate of water into each WGS is calculated using energy balance between enthalpy of the gas flows, enthalpy of the flow at the desired temperature, and the heat of water vaporization. It is assumed that the PROX air injection, $W_{a i r}^{\text {prox }}$, is scheduled based on the stack current at the value twice needed (Brown, 2001; Doss et al., 2001) at the designed operating condition.

\subsection{Anode (AN)}

Mass conservation is used to model the pressure dynamics in the anode volume. To simplify the model, only three mass flows are considered, including flows into and out of the anode volume and the rate of hydrogen consumed in the fuel cell reaction. The dynamic equations are

$$
\frac{d p^{a n}}{d t}=\frac{R T_{a n}}{M_{a n} V_{a n}}\left(W^{w r x}-W^{a n}-W_{H_{2}, \text { react }}\right)
$$


$\frac{d p_{H_{2}}^{a n}}{d t}=\frac{R T_{a n}}{M_{H_{2}} V_{a n}}\left(x_{H_{2}}^{w r x} W^{w r x}-x_{H_{2}}^{a n} W^{a n}-W_{H_{2}}\right.$, react $)$

where $W^{a n}$ is calculated as a function of the anode pressure, $p^{a n}$, and the ambient pressure, $p_{a m b}$, using Equation (5). The rate of hydrogen reacted is a function of stack current, $I_{s t}$, through the electrochemistry principle (Larminie and Dicks, 2000)

$$
W_{H_{2}}, \text { react }=M_{H_{2}} \frac{n I_{s t}}{2 F}
$$

where $n$ is the number of fuel cells in the stack and $F$ is the Faraday's number (96485 coulombs).

Two meaningful variables which are hydrogen utilization, $U_{\mathrm{H}_{2}}$, and anode hydrogen mole fraction, $y_{\mathrm{H}_{2}}$, can be calculated by

$$
U_{H_{2}}=\frac{\mathrm{H}_{2} \text { reacted }}{\mathrm{H}_{2} \text { supplied }}=\frac{W_{H_{2}}, \text { react }}{x_{H_{2}}^{w x} W^{w r x}}
$$

and $y_{H_{2}}=\frac{p_{H_{2}}^{a n}}{p^{a n}}$.

\section{SIMULATION AND MODEL VALIDATION}

The low-order (10 states) model described in the previous sections is developed in MATLAB/Simulink platform. The model is parameterized and validated with the results of a high-order ( $>300$ states) detailed model (Eborn et al., 2003). The detailed model includes spatial variation and exact chemical reaction rates for all the species. The detailed model is developed using Dymola software (Tiller, 2001) and is imported as an S-function in Simulink. The two models are compared with equivalent inputs. The model parameters used are for $200 \mathrm{~kW}$ fuel cell system which is a power range needed for a bus or a heavy-duty vehicle propulsion system. The focus of our work is to capture the essential dynamic input/output behavior, and, thus our main focus is reasonable agreement of transient responses. The FPS key performance variables are the $\mathrm{O} 2 \mathrm{C}$ ratio, the $\mathrm{CPOX}$ temperature, the FPS exit total flow rate, and the FPS exit hydrogen flow rate. Several parameters, such as the orifice constants and the component volumes, are adjusted appropriately in order to obtain comparable transient responses.

The nominal operating point used in the validation is chosen at the oxygen to carbon ratio $\lambda_{O 2 C}=0.6$ and the stack hydrogen utilization $U_{H_{2}}=80 \%$ (Doss et al., 2001). Step changes (up/down) of the three inputs: the stack current, $I_{s t}$, the blower signal, $u_{b l o}$, and the fuel valve signal, $u_{\text {valve }}$, are applied individually at time 400, 800, and 1200 seconds, respectively, followed by the simultaneous step changes of all inputs at 1600 seconds. The responses of the key variables are shown in Figure 4.

It can be seen that, despite the offset, there is a good agreement between the two models for most transient responses. The model is also tested at different power (current) operations and transient responses also agree well. A more accurate model can be developed
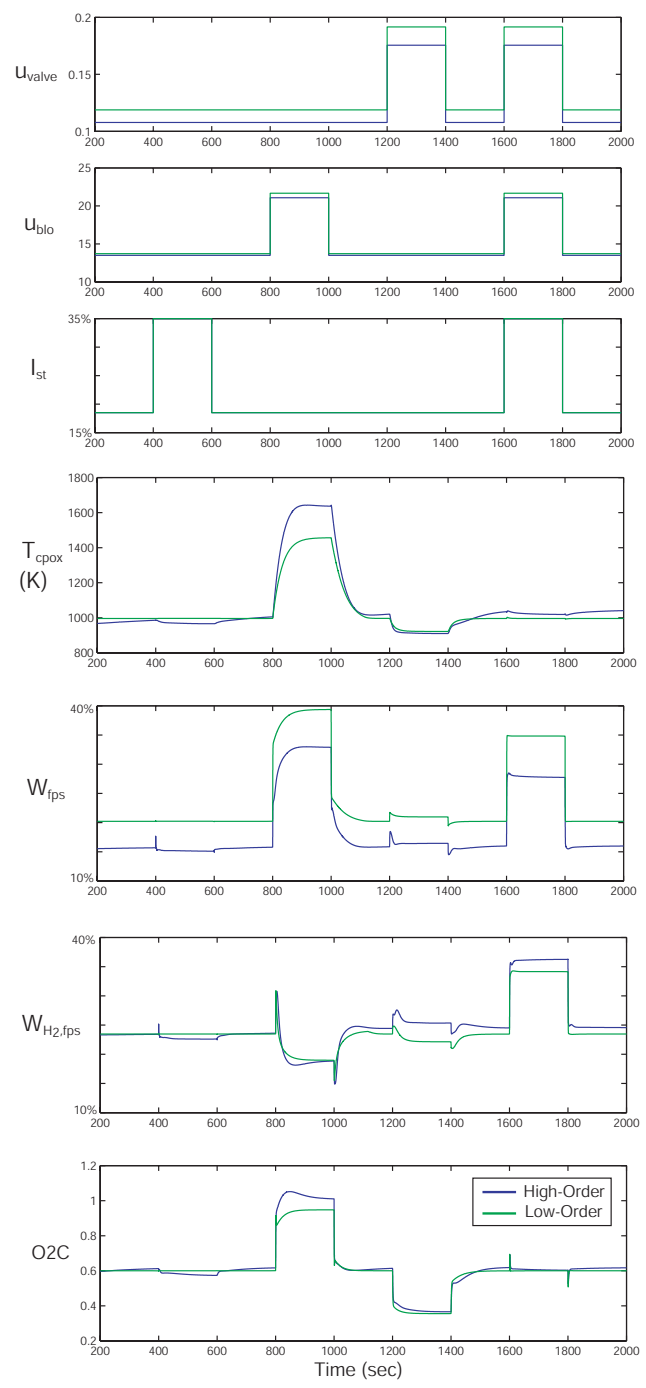

Fig. 4. Model Validation Results: Inputs and Performance Variables. Blue (Dark) = high-order model; green $($ light $)=$ low-order model

with the expense of extra complexity and/or higher system order.

\section{OBSERVABILITY ANALYSIS}

The dynamic FPS model is useful for control analysis and design. Here, we illustrate the use of the model to develop a plant estimator to be used for plant estimation and diagnostics. The model can be used to develop real-time observers to estimate critical stack variables that may be hard to measure or augment existing stack sensors for redundancy in fault detection (Glass, 2000). Linear observability analysis can provide an indication of useful measurements during the preliminary design phase.

We first assume perfect measurements of $T_{c p x}$ and $y_{H_{2}}$. The linearized open loop system becomes 


$$
\begin{aligned}
& \dot{x}=A x+B_{u} u+B_{w} w \\
& z=C_{z} x+D_{z u} u+D_{z w} w .
\end{aligned}
$$

where $w=I_{s t}, u=\left[u_{\text {blo }} u_{\text {valve }}\right]^{T}$ and $z=\left[T_{c p x} y_{H_{2}}^{a n}\right]$. The observability matrix $O\left(A, C_{z}\right)$ has full rank and thus it is possible to build the estimator using only these two measurements. However, the condition number of the observability gramian, $Q_{o b s}$, i.e. solution of $A^{T} Q_{o b s}+Q_{o b s} A=-C_{z}^{T} C_{z}$, is high. To better evaluate the system observability we normalize the observability gramian $\left(N Q_{o b s}\right)$

$$
N Q_{o b s}=\frac{\operatorname{cond}\left(Q_{o b s},\left\{C=C_{z}\right\}\right)}{\operatorname{cond}\left(Q_{o b s},\{C=I\}\right)}=2 \times 10^{5} .
$$

Large normalized observability gramian indicates that $\left(A, C_{z}\right)$ pair is weakly observable.

In practice, the CPOX temperature measurement and anode hydrogen mole fraction can not be instantaneously measured. The temperature and hydrogen sensors are normally slow, with time constants of approximately 60 seconds and 20 seconds, respectively. The lag in the measurements degrades further the estimator performance.

Each set of measurements provides different degree of observability as can be seen by comparing the normalized condition number of the observability gramian in Table 1 . The lag contributed by the sensors significantly degrades the system observability. However, adding the fuel and air flow measurements lowers the observability condition number to a value lower than the one obtained with perfect measurement of $T_{c p x}$ and $y_{H_{2}}$. We can, thus, expect a better estimation performance. Even better estimation can be expected if additional measurements such as anode pressure are available as shown in the table below. More work is needed to define the critical measurements that will be beneficial for the combined observer-based controller.

Table 1. Normalized condition number of observability gramian

\begin{tabular}{|c|c|}
\hline Measurements & Condition Number \\
\hline$T_{c p x}, y_{H_{2}}$ & $2 \times 10^{5}$ \\
$T_{c p x}^{m}, y_{H_{2}}^{m}$ & $1.3 \times 10^{10}$ \\
$T_{c p x}^{m}, y_{H_{2}}^{m}, W_{\text {air }}, W_{\text {fuel }}$ & 3672.7 \\
$T_{c p x}^{m}, y_{H_{2}}^{m}, W_{\text {air }}, W_{\text {fuel }}, p^{m i x}$ & 1928.8 \\
\hline
\end{tabular}

\section{CONCLUSION}

A low-order (10 states) nonlinear model of the FPS is developed with a focus on the dynamic behaviors associated with the flow and the pressures in the FPS, the temperature of the CPOX and the hydrogen generation for a fuel cell. The model is based on physical parameters of the plant and can be easily scaled to represent any partial oxidation-based FPS. The FPS model is parameterized and validated against a high-order ( $>300$ states) fuel cell system model, which was validated against experimental data. The transient behaviors of the low-order model agree well with that of the high-order model. We present observability analysis that can help in measurement/sensor selection.

\section{REFERENCES}

Ahmed, S. and M. Krumpelt (2001). Hydrogen from hydrocarbon fuels for fuel cells. International Journal of Hydrogen Energy 26, 291-301.

Birch, S. (2001). Ford's focus on the fuel cell. Automotive Engineering International pp. 25-28.

Brown, L.F. (2001). A comparative study of fuels for on-board hydrogen production for fuel-cell-powered automobiles. International Journal of Hydrogen Energy 26, 381-397.

Dicks, A.L. (1996). Hydrogen generation from natural gas for the fuel cell systems of tomorrow. Journal of Power Sources 61, 113-124.

Doss, E.D., R. Kumar, R.K. Ahluwalia and M. Krumpelt (2001). Fuel processors for automotive fuel cell systems: a parametric analysis. Journal of Power Sources 102, 115.

Eborn, J., L.M. Pedersen, C. Haugstetter and S. Ghosh (2003). System level dynamic modeling of fuel cell power plants. Proceedings of the 2003 American Control Conference pp. 2024-2029.

Gardner, T.H., D.A. Berry, K.D. Lyons, S.K. Beer and A.D. Freed (2002). Fuel processor integrated $\mathrm{H}_{2} \mathrm{~S}$ catalytic partial oxidation technology for sulfur removal in fuel cell power plants. Fuel 81, 2157-2166.

Glass, R.S. (2000). Sensor needs and requirements for proton exchange membrane fuel cell systems and direct-injection engines. Technical report. Department of Energy. Published by Lawrence Livermore National Laboratory.

Larentis, A.L., N.S. de Resende, V.M.M. Salim and J.C. Pinto (2001). Modeling and optimization of the combined carbon dioxide reforming and partial oxidation of natural gas. Applied Catalysis 215, 211-224.

Larminie, James and Andrew Dicks (2000). Fuel Cell Systems Explained. John Wiley \& Sons Inc. West Sussex, England.

Ledjeff-Hey, K., J. Roses and R. Wolters (2000). $\mathrm{CO}_{2}$ scrubbing and methanation as purification system for PEFC. Journal of Power Sources 86, 556-561.

Pukrushpan, Jay Tawee (2003). Modeling and Control of Fuel Cell Systems and Fuel Processors. PhD thesis. University of Michigan.

Pukrushpan, J.T., A.G. Stefanopoulou and H. Peng (2002). Modeling and control issues of PEM fuel cell stack system. Proceedings of the 2002 American Control Conference pp. 3117-3122.

Song, R-H, C-S Kim and D.R. Shin (2000). Effects of flow rate and starvation of reactant gases on the performance of phosphoric acid fuel cells. Journal of Power Sources 86, 289-293.

Thomas, C.E., B.D. James, F.D. Lomax Jr and I.F. Kuhn Jr (2000). Fuel options for the fuel cell vehicle: hydrogen, methanol or gasoline?. International Journal of Hydrogen Energy 25, 551-567.

Tiller, Michael (2001). Introduction to Physical Modeling with Modelica. Kluwer Academic Publishers. Boston.

Zhu, J., D. Zhang and K.D. King (2001). Reforming of $\mathrm{CH}_{4}$ by partial oxidation: thermodynamic and kinetic analyses. Fuel 80, 899-905. 